\title{
Drug Liver Injury Induced by Olmesartan Mediated by Autoimmune-Like Mechanism: A Case Report
}

\author{
Manuel de la Torre-Aláez ${ }^{1}$, Mercedes Iñarrairaegui ${ }^{2}$ \\ ${ }^{1}$ Liver Unit, Clínica Universidad de Navarra, Madrid, Spain \\ 2 Liver Unit, Clínica Universidad de Navarra, Pamplona, Spain
}

Received: 27/11/2019

Accepted: 30/11/2019

Published: 03/01/2020

\begin{abstract}
How to cite this article: de la Torre-Aláez M, Iñarrairaegui M. Drug liver injury induced by olmesartan mediated by autoimmune-like mechanism: a case
\end{abstract} report. EJCRIM 2020;7: doi:10.12890/2020_001407.

Conflicts of Interests: The Authors declare that there are no competing interest

Acknowledgements: We appreciate the histological images provided by Dra. Marta Abengozar from the Department of Pathology of Clínica Universidad de Navarra in Pamplona.

This article is licensed under a Commons Attribution Non-Commercial 4.0 License

\section{ABSTRACT}

Autoimmune hepatitis $(\mathrm{AlH})$ was the first liver disease for which an effective therapeutic intervention was carried out, using prednisolone; its usefulness was demonstrated in several clinical trials. Nevertheless, AlH still remains a difficult diagnosis in some cases, because it is necessary to dismiss other possible diagnoses, and perhaps due to it being a heterogeneous disease. The relationship between drug-induced liver injury (DILI) and AIH is complex and not fully understood. There are three possible scenarios: (1) DILI with a strong immunoallergic component mimicking AIH; (2) AlH mimicking a DILI due to drug exposure and (3) AlH triggered by exposure to an offending drug (druginduced AlH). Drug-induced AlH is well described and documented for some drugs such as nitrofurantoin and minocycline. Histologically distinguishing DILI from AlH remains a challenge. We present an interesting case report which met serologic criteria and histological confirmation to establish $\mathrm{AlH}$, but discontinuation of a suspected drug resolved hypertransaminasaemia.

\section{LEARNING POINTS}

- Idiosyncratic drug-induced liver injury is one of the most challenging liver disorders.

- Diagnosis of drug-induced liver injury is a complex question; this can evolve to severe hepatotoxicity if it is not diagnosed promptly.

- Usually, olmesartan and similar anti-hypertensive drugs are not considered drugs with the potential to cause liver damage.

\section{KEYWORDS}

Olmesartan, drug-induced liver injury, autoimmune-like mechanism, hypertransaminasaemia

\section{CASE DESCRIPTION}

The patient was an 80-year-old woman with a history of hypertension being treated with olmesartan/amlodipine since 2015, dyslipidaemia, sigmoid diverticulosis and serous papillary peritoneal adenocarcinoma diagnosed on November 2015, treated by surgery and chemotherapy with carboplatin-paclitaxel, receiving 6 cycles (the last cycle was received in June 2016). A complete response was confirmed in December 2016, with tumour markers within the normal range and no findings suggestive of neoplasm in PET-CT.

In July 2017 the patient was referred to the Liver Unit due to alterations found during liver tests: aspartate aminotransferase (AST) 207 IU/L $(\mathrm{N}<33$ ), alanine aminotransferase (ALT) $213 \mathrm{IU} / \mathrm{L}(\mathrm{N}<33)$, gamma-glutamyl transpeptidase (GGTP) $21 \mathrm{IU} / \mathrm{L}$ ( $\mathrm{N}<40$ ), alkaline phosphatase (ALP) $116 \mathrm{IU} / \mathrm{L}(\mathrm{N}<105)$, total bilirubin $0.5 \mathrm{mg} / \mathrm{dl}(\mathrm{N}<1.2)$ and international normalized ratio 1.2 . 
These findings were discovered during follow-up of the tumoural disease. No tumour marker elevation or CT alterations were observed. Liver tests were normal before starting treatment with olmesartan. The patient did not take herbal products, or other potentially hepatotoxic drugs. She had not travelled outside Europe recently. She had no history of abusive alcohol consumption and her body mass index was 26.6 $\mathrm{kg} / \mathrm{m}^{2}$. Physical examination was normal.

No fever, rash, eosinophilia, jaundice or coagulopathy was observed during follow-up, and she did not require hospitalization during the course of the disease.

We repeated the liver tests 1 week later, with alterations persisting at 5 times the upper limit of normal values. An extensive evaluation was carried out, which ruled out serological viral hepatitis (hepatitis A, B and C) and metabolic liver disease, as well as Wilson disease or haemochromatosis; there was a normal blood count, normal gamma globulin, thyroid and lipid profiles, and no other relevant biochemistry alterations. Testing for antinuclear antibodies (ANA) was positive with a higher titre of 1/2,560 and a homogeneous pattern; testing for ASMA, anti-LKM1 antibodies and AMA was negative.

In view of these findings and the persistent alterations seen in liver tests, we conducted a liver biopsy that showed the preserved architecture of liver parenchyma, with a portal polymorphic inflammatory infiltrate with occasional plasma cells (Fig. 1), lymphoplasmacytic patchy infiltrate in the lobule and mild portal fibrosis (Fig. 2). Occasional nuclear pseudoinclusions in hepatocytes were found. No signs suggestive of viral aetiology, abnormal iron deposits or Mallory's hyaline were found with the corresponding techniques. Based on those results, we decided to stop olmesartan but without introducing prednisolone or other immunosuppressive therapy.

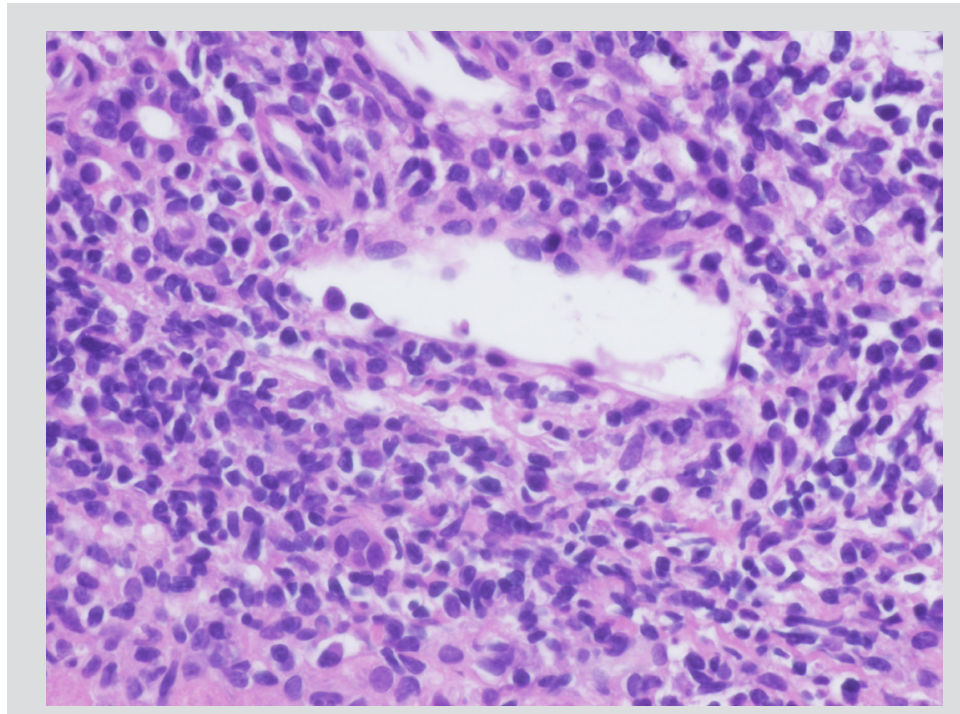

Figure 1. Histological results from liver biopsy showing an inflammatory infiltrate in the portal space with some plasmatic cells. These are the typical findings in patients with autoimmune hepatitis (images have been provided by Dra. M. Abengozar, Department of Pathology, Clínica Universidad de Navarra)

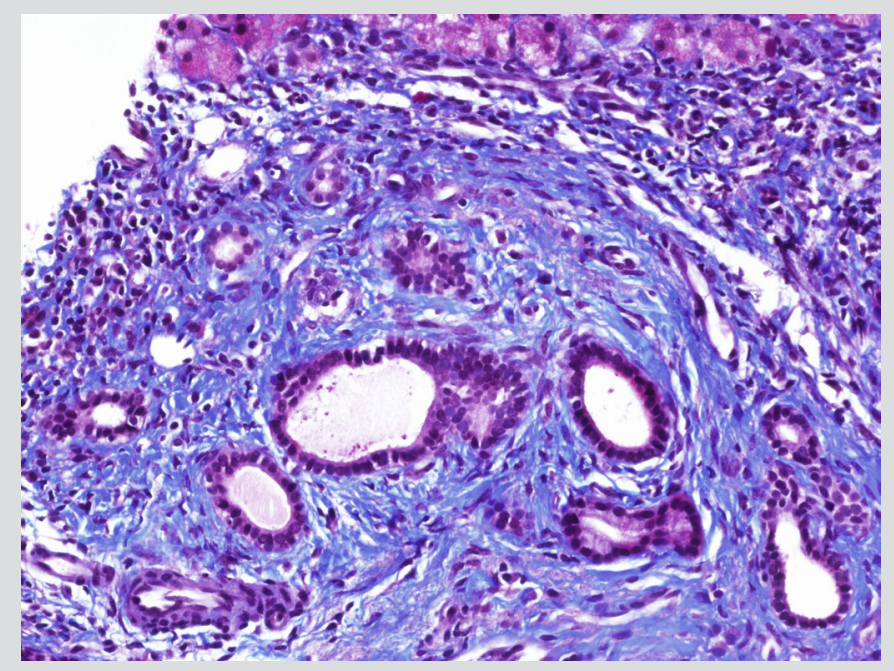

Figure 2. Histological results from liver biopsy showing portal fibrosis using Masson's trichrome stain (images have been provided by Dra. M. Abengozar, Department of Pathology, Clínica Universidad de Navarra)

Two month after olmesartan withdrawal, liver tests, were decreased to the normal range (ALT 20 IU/L, AST 25 IU/L, GGTP 16 IU/L and ALP $106 \mathrm{IU} / \mathrm{L})$. One year after, liver tests were still normal; the patient remains asymptomatic as shown in Fig. 3.

\section{DISCUSSION}

Treatment of arterial hypertension with olmesartan has been associated with the development of sprue-like enteropathy, characterized by diarrhoea, malabsorption, weight loss and varying degrees of duodenal mucosal atrophy ${ }^{[1-4]}$. Ianiro et al. ${ }^{[4]}$ reviewed the literature and found 11 publications, for an overall number of 54 patients, who had developed sprue-like enteropathy associated with olmesartan. The duration of treatment was 3.3 years. Testing for anti-transglutaminase and anti-endomysial antibodies was found to be negative. The presence of anti-enterocyte antibodies was tested in 21 patients, with a positive result in only 3 patients. After discontinuation of olmesartan, clinical remission occurred in $100 \%$ of patients. 


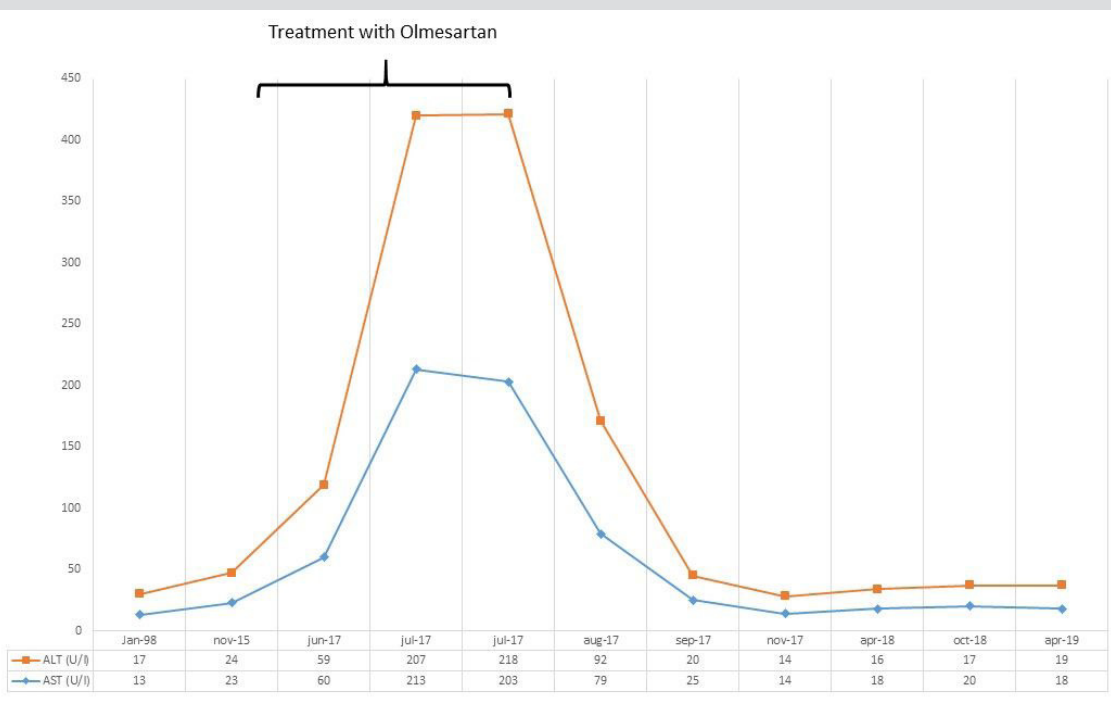

Figure 3. Evolution of liver test results (AST/GOT and ALT/GPT). This shows

The underlying physiopathological mechanisms of olmesartan-associated enteropathy are unknown, although several studies suggest that cell-mediated immunity damage may play a role ${ }^{[1,4]}$.

The FDA communicated the risk of enteropathy induced by olmesartan in July 2013. Although an association with enteropathy is more common with olmesartan, enteropathy with other angiotensin II receptor blockers (ARBs) has been reported ${ }^{[2]}$.

The first report we found in the literature regarding the implications of autoimmune-like chronic hepatitis mediated by treatment with olmesartan was published by Barge et al. ${ }^{[5]}$ in April 2017. These authors described a 61-year-old woman with anorexia, asthenia and weight loss of $10 \mathrm{~kg}$ without diarrhoea, with abnormal liver test results and ANA-positive titres. Elastography and biopsy results were compatible with fibrosis and autoimmune hepatitis. Three months after olmesartan withdrawal, the clinical and analytical evolution was favourable. Treatment with immunosuppressive drugs was not started. A second biopsy was performed, showing regression of fibrosis and resolution of interface hepatitis. In this case, it was suggested that the mechanism of hepatic injury was autoimmune, on the basis of the findings and reports in the literature with respect to enteropathy mediated by immunity damage.

Idiosyncratic drug-induced liver injury is 1 of the most challenging liver disorders faced by hepatologists that has been known. An important effort exists to understand the mechanism of liver damage and to identify drugs or other potential toxic agents due to the frequency this is seen in clinical practice. Even some scientific societies are developing new recommendations on this issue. For example, it's worth noting the efforts from the European Association for the Study of the Liver (EASL), which published its new guidelines this year to summarize the available scientific evidence ${ }^{[6]}$.

It is accepted that the diagnosis of drug-induced liver injury is a complex question and this can evolve to a severe hepatotoxicity if it is not diagnosed promptly. For that, we think it is important for clinicians to know potential liver-toxic drugs.

Our case report is an interesting liver toxicity case due to an anti-hypertensive drug used in clinical practice and the possible immunomediated mechanism of damage associated with this. Usually, olmesartan and similar anti-hypertensive drugs are not considered to be drugs with the potential for liver damage.

We attempt to demonstrate this relationship with liver test result evolution after withdrawal of the drug, autoimmune serologic testing and pathologic findings. Hypertransaminasaemia was resolved without the need for treatment with corticosteroids or other immunosuppressive drugs. We also propose once we have reviewed our case, based on analytical evolution, serological data and liver biopsy, that an autoimmune mechanism may play a role in liver damage. In our case, the clinical manifestations were not as evident as in the case of Barge et al. ${ }^{[5]}$.

In our opinion, treatment with olmesartan should be considered in the case of a patient with unexplained mild to moderate hypertransaminasaemia and serologic data suggestive of an autoimmune phenomenon with no other signs suggestive of autoimmune hepatitis, once other aetiological causes and other toxic agents have been excluded. We think that this case will be of interest to clinicians who usually attend patients with abnormal liver test results such as internists or hepatologists. 


\section{REFERENCES}

1. Rubio-Tapia A, Herman M, Ludvigsson JF, Kelly DG, Mangan TF, Wu TT, Murray JA. Severe Spruelike enteropathy associated with olmesartan. Mayo clinic proceedings 2012 Aug;87(8):732-8.

2. Basson M, Mezzarobba M, Weill A, Ricordeau P, Allemand H, Alla F, et al. Severe intestinal malabsorption associated with olmesartan: a French nationwide observational cohort study. Gut 2016;65(10):1664-1669.

3. Naik D, Martelli M, Hernandez Gonzalo D, Sharma A, Pannu D. An atypical case of chronic diarrhoea: olmesartan-induced sprue-like enteropathy. BMJ Case Rep 2015;2015:bcr2015212318

4. Ianiro G, Bibbo S, Montalto M, Ricci R, Gasbarrinina A, Cammarota G. Systematic review: Sprue-like enteropathy associated with olmesartan. Aliment Pharmacol Ther Syst 2014;40:16-23.

5. Barge S, Ziol M, Nault J. Autoimmune-like chronic hepatitis induced by olmesartan. Hepatology 2017;66:2086-2088

6. European Association for the Study of the Liver. EASL Clinical Practice Guidelines: drug-induced liver injury. J Hepatol 2019;70(6):1222-1261. logical results from liver biopsy. 